\title{
UV and X-ray observations of mass outflows in AGN
}

\author{
Gerard A. Kriss ${ }^{1}$ \\ ${ }^{1}$ Space Telescope Science Institute, 3700 San Martin Drive, Baltimore, MD 21218, USA \\ email: gak@stsci.edu
}

\begin{abstract}
More than half of all low-redshift AGN exhibit UV and X-ray absorption by highly ionized gas. The observed UV and X-ray absorption lines are almost aiways blue-shifted at velocities of hundreds of $\mathrm{km} \mathrm{s}^{-1}$, indicating that the absorbing gas is outflowing from the active nucleus. In some cases the inferred mass flux rivals the Eddington limit of the central black hole, an indication that these outflows are intimately related to the mass accretion and energy generation mechanism in AGN. The ejected material can also have an affect on the interstellar medium of the host galaxy and the surrounding intergalactic medium. Over the past several years, coordinated UV and X-ray observations of several bright AGN at high spectral resolution using HST, FUSE, Chandra, and XMM-Newton have contributed greatly to our understanding of these outflows. I will give an overview of these recent observations, summarize our FUSE survey of low-redshift AGN, and interpret the results in the context of models of winds from accretion disks and thermally driven winds from the obscuring torus.
\end{abstract}

\section{Introduction}

Mass outflows from active galactic nuclei (AGN) can profoundly affect the evolution of the central engine (Blandford \& Begelman 1999), the host galaxy and its interstellar medium (Silk \& Rees 1998; Wyithe \& Loeb 2003) and also the surrounding intergalactic medium (IGM) (Cavaliere, Lapi \& Menci 2002; Granato et al. 2004; Scannapieco \& Oh 2004). Winds from the high metal-abundance nuclear regions may be a significant source for enriching the IGM (Adelberger et al. 2003). Absorption by the outflow can also collimate the ionizing radiation (Kriss et al. 1997) and thereby influence the ionization structure of the host galaxy and the surrounding IGM.

The outflowing gas in AGN is sometimes visible as extended, bi-conical emission at visible or X-ray wavelengths (e.g., NGC 4151, Evans et al. 1993, Hutchings et al. 1998; NGC 1068, Ogle et al. 2003), but it most frequently manifests itself as blue-shifted absorption features in their UV and X-ray spectra. About half of all low-redshift AGN show X-ray absorption by highly ionized gas (Reynolds 1997; George et al. 1998), and a similar fraction show associated UV absorption in ionized species such as C IV (Crenshaw et al. 1999) and O VI (Kriss 2001). In more luminous quasars, the fraction of objects in the Sloan Digital Sky Survey that shows broad C IV absorption troughs rises steeply as the troughs become narrower (Tolea, Krolik \& Tsvetanov 2002; Reichard et al. 2003), comprising over $30 \%$ of the quasar population at widths narrower than $1000 \mathrm{~km} \mathrm{~s}^{-1}$. For AGN that have been observed in both the X-ray and the UV, there is a one-toone correspondence between objects showing X-ray and UV absorption, implying that the phenomena are related in some way (Crenshaw et al. 1999). The high frequency of occurrence of UV and X-ray absorption suggests that the absorbing gas has a high covering fraction, and that it is present in all AGN. The gas has a total mass exceeding $\sim 10^{3} \mathrm{M}_{\odot}$ (greater than the broad-line region, or BLR), and is outflowing at a rate $>0.1 \mathrm{M}_{\odot} \mathrm{yr}^{-1}(10 \times$ the accretion rate in some objects) (Reynolds 1997). 
Key questions for understanding the outflowing, absorbing gas in AGN are:

- What are the column densities and ionic abundances in the absorbing gas?

Even such a basic question is uncertain since the absorption line profiles are complex. Doublet ratios show that the absorbers can be optically thick, but they are not black at line center. Thus column densities are frequently underestimated, sometimes by as much as an order of magnitude (Arav, Korista \& de Kool 2002; Arav et al. 2003).

- Where is the absorbing gas located?

The location of the absorbers is a vital clue to the process producing the outflow. Winds arising from an accretion disk (Königl \& Kartje 1994; Murray et al. 1995; Elvis 2000; Proga 2000) will have material at a broad range of radii reaching from near the disk itself to beyond the BLR. Thermally driven winds arising from the obscuring torus (Krolik \& Kriss 1995, 2001) will lie at much larger radii, typically at distances of $\sim 1$ pc. The radial location also determines the mass flux - the larger the distance, the higher the total mass and the mass flux in the wind.

- Do variations reflect an ionization response, or are they due to bulk motion?

In the limited number of monitoring campaigns carried out thus far, examples of both have been seen. The neutral hydrogen and the C III absorption in NGC 4151 responds quite clearly to continuum variations (Kriss et al. 1997; Espey et al. 1998). Some absorbing clouds in NGC 3783 have shown changes consistent with an ionization response, while others appear to be due to bulk motion (Crenshaw et al. 1999; Gabel et al. 2003b).

- How are the X-ray and UV absorption related?

In some cases, UV absorbing gas may be directly associated with the X-ray warm absorber (3C351: Mathur et al. 1994; NGC 5548: Mathur, Wilkes \& Elvis 1995; NGC 3516: Kraemer et al. 2002). In the extensive recent Chandra/FUSE/HST campaign on NGC 3783 (Kaspi et al. 2002; Gabel et al. 2003a), the kinematics of the X-ray absorbing gas are also a good match to the UV-absorbing gas. In other cases, however, the UV gas appears to be in an even lower ionization state, and there is no direct relation between the X-ray absorption and the multiple kinematic components seen in the UV (NGC 4151: Kriss et al. 1995, Kraemer et al. 2001; NGC 3516: Kriss et al. 1996a,b; NGC 5548: Mathur, Elvis \& Wilkes 1999, Crenshaw et al. 2003; Mrk 509: Kriss et al. 2000b, Yaqoob et al. 2003; NGC 7469: Kriss et al. 2000a, 2003; Blustin et al. 2003). The X-ray absorbing gas itself contains material spanning a large range of ionization parameters (Lee et al. 2002; Sako et al. 2003; Netzer et al. 2003), and it is likely that this broad range of physical conditions can also include the UV-absorbing ions. This is a natural prediction of the thermally driven wind model of Krolik \& Kriss (1995, 2001), and would also be likely in disk-driven winds.

To address some of these questions, we have been conducting a survey of the $\sim 100$ brightest AGN using the Far Ultraviolet Spectroscopic Explorer (FUSE). The short wavelength response (912-1187 $\AA$ ) of FUSE (Moos et al. 2000) enables us to make highresolution spectral measurements $(R \sim 20,000)$ of the high-ionization ion $\mathrm{O}$ VI and the high-order Lyman lines of neutral hydrogen. As of November 1, 2002, we have observed a total of $87 \mathrm{AGN}$. Of these, 57 have $z<0.15$, so that the O vI doublet is visible in the FUSE band. The $\mathrm{O}$ VI doublet is a crucial link between the higher ionization absorption edges seen in the X-ray and the lower ionization absorption lines seen in earlier UV observations. The high-order Lyman lines provide a better constraint on the total neutral hydrogen column density than Ly $\alpha$ alone. Lower ionization species such as $\mathrm{C}$ III and $\mathrm{N}$ III also have strong resonance lines in the FUSE band, and these often are useful for setting constraints on the ionization level of any detected absorption. The Lyman and Werner bands of molecular hydrogen also fall in the FUSE band, and we have searched 

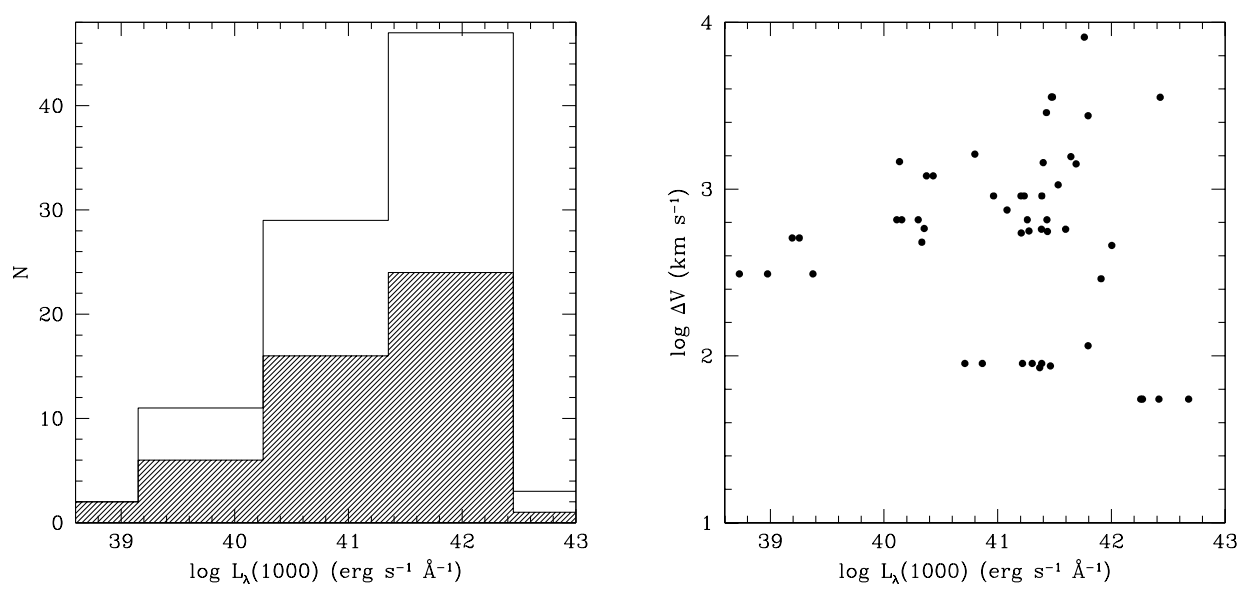

Figure 1. Left: Histogram of FUSE AGN versus luminosity. The shaded area shows the number of objects exhibiting intrinsic absorption. Right: The points show outflow velocity as a function of luminosity.

for (but not found) intrinsic $\mathrm{H}_{2}$ absorption that may be associated with the obscuring torus.

\section{Survey Results}

As shown in the left panel of Figure 1, absorption is common at all luminosities, and over $50 \%$ (30 of 53) of the low-redshift Type 1 AGN observed using FUSE show detectable O VI absorption, comparable to those Seyferts that show longer-wavelength UV (Crenshaw et al. 1999) or X-ray (Reynolds 1997; George et al. 1998) absorption. None show intrinsic $\mathrm{H}_{2}$ absorption. We see three basic morphologies for $\mathrm{O}$ VI absorption lines: (1) Single: 13 of 30 objects exhibit single, narrow, isolated O VI absorption lines, as illustrated by the spectrum of Ton S180 (Turner et al. 2001). PG0804+761, shown in the top panel of Figure 2, is another example. (2) Blend: multiple O vi absorption components that are blended together. Ten of 30 objects fall in this class, and the spectrum of Mrk 279 is typical (Scott et al. 2004). The middle panel of Figure 2 shows Mrk 478 as another example. (3) Smooth: The 7 objects here are an extreme expression of the "blend" class, where the O VI absorption is so broad and blended that individual O VI components cannot be identified. NGC 4151 typifies this class (Kriss et al. 1992, 1995; Kriss 2001). The mini-BAL QSO PG1411+442 is another example of this class, shown in the bottom panel of Figure 2.

Individual $\mathrm{O}$ vi absorption components in our spectra have FWHM of $50-750 \mathrm{~km} \mathrm{~s}^{-1}$, with most objects having FWHM $<100 \mathrm{~km} \mathrm{~s}^{-1}$. The multiple components that are typically present are almost always blue shifted, and they span a velocity range of 200 $4000 \mathrm{~km} \mathrm{~s}^{-1}$; half the objects span a range of $<1000 \mathrm{~km} \mathrm{~s}^{-1}$. As shown in the right panel of Figure 1, the maximum outflow velocities show a tendency to increase with source luminosity, perhaps indicating that radiative acceleration plays some role in the dynamics. Note also that there is a population of low-velocity absorbers present at all luminosities. 


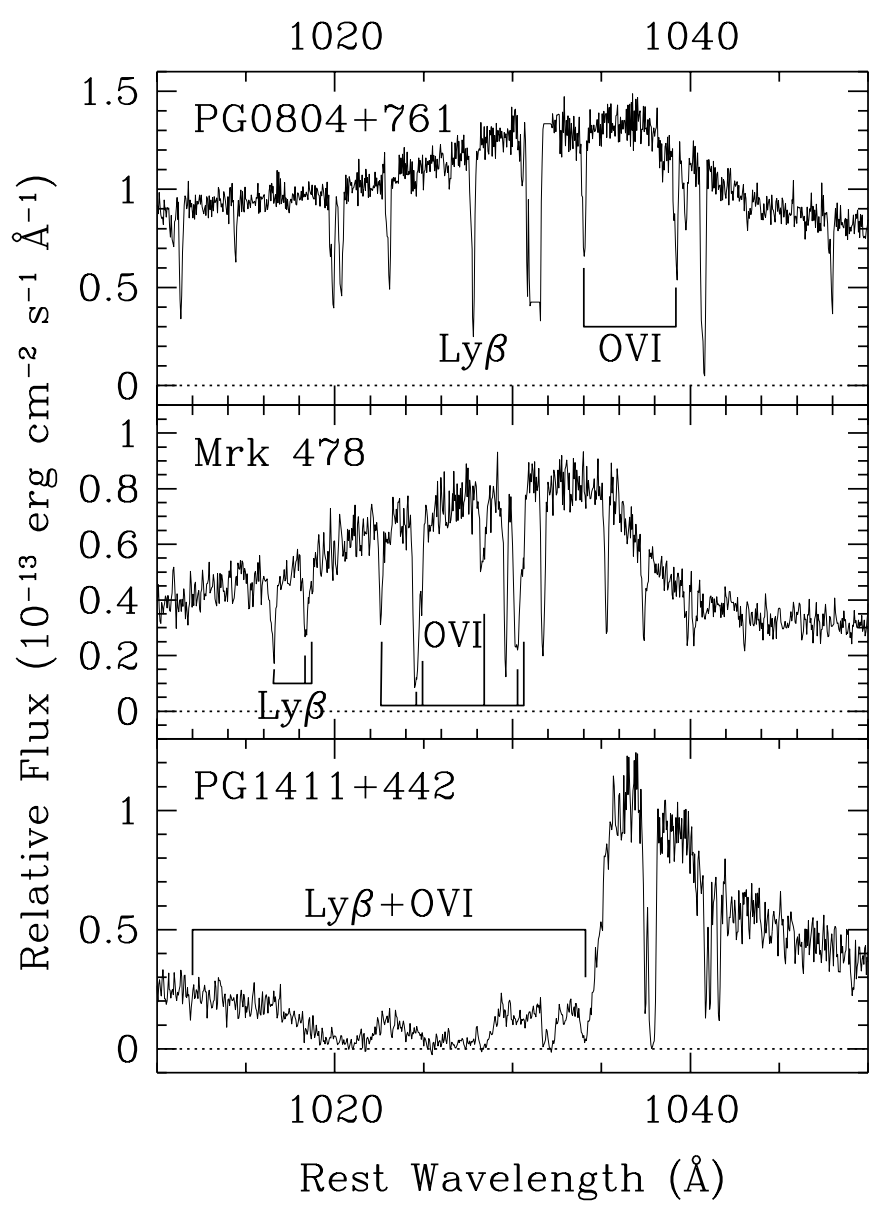

Figure 2. The three classes of O VI absorption line morphology: Single, isolated lines: PG 0804+761 (top); Multiple, blended lines: Mrk 478 (middle); Broad, blended trough: PG1411+442 (bottom).

\section{Discussion}

The multiple kinematic components frequently seen in the UV absorption spectra of AGN clearly show that the absorbing medium is complex, with separate UV and X-ray dominant zones. In some cases, the UV absorption component corresponding to the Xray warm absorber can be clearly identified (e.g., Mrk 509, Kriss et al. 2000b). In others, however, no UV absorption component shows physical conditions characteristic of those seen in the X-ray absorber (NGC 3516, Kriss et al. 1996a,b; NGC 5548, Brotherton et al. 2002). One potential geometry for this complex absorbing structure is high-density, lowcolumn UV-absorbing clouds embedded in a low-density, high-ionization medium that dominates the X-ray absorption.

Disk-driven winds are a possible explanation for some cases of AGN outflows. By analogy to stellar winds, one would expect the terminal velocity of an AGN outflow to reflect the gravity of its origin. Disk-driven winds should therefore have velocities in the range of several thousand $\mathrm{km} \mathrm{s}^{-1}$. Objects with broad, smooth profiles might fall in this category. The geometry proposed by Elvis (2000) suggests that these objects should have only modest inclinations. However, two prime examples of Seyferts with broad smooth 
absorption troughs, NGC 3516 (Hutchings et al. 2001) and NGC 4151 (Kriss 2001), are likely the highest inclination sources in our sample, given their extended, bi-conical narrow emission-line region morphologies (Miyaji, Wilson \& Perez-Fournon 1992; Evans et al. 1993) and their opaque Lyman limits (Kriss et al. 1997).

The lower velocities we observe in objects like NGC 3783 and NGC 5548 are more compatible with thermally driven winds from the obscuring torus (Krolik \& Kriss 1995, 2001). In these thermally driven winds, photoionized evaporation in the presence of a copious mass source (the torus) locks the ratio of ionizing intensity to total gas pressure (the ionization parameter $\Xi$ ) at a critical value. For AGN spectral energy distributions lacking a strong extreme ultraviolet bump, such as the composite spectra of quasars assembled by Zheng et al. (1997), Laor et al. (1997), and Telfer et al. (2002), the ionization equilibrium curve exhibits an extensive vertical branch. Thus, at the critical ionization parameter for evaporation, there is a broad range of temperatures that can coexist in equilibrium at nearly constant pressure. For this reason, the flow is expected to be strongly inhomogeneous. Outflow velocities are typical of the sound speed in the heated gas, or several hundred $\mathrm{km} \mathrm{s}^{-1}$, comparable to the velocities seen in many AGN.

In summary, we find that O VI absorption is common in low-redshift $(z<0.15)$ AGN. Thirty of 53 Type 1 AGN with $z<0.15$ observed using FUSE show multiple, blended O vi absorption lines with typical widths of $\sim 100 \mathrm{~km} \mathrm{~s}^{-1}$ that are blueshifted over a velocity range of $\sim 1000 \mathrm{~km} \mathrm{~s}^{-1}$. Those galaxies in our sample with existing X-ray or longer wavelength UV observations also show C IV absorption and evidence of a soft Xray warm absorber. In some cases, a UV absorption component has physical properties similar to the X-ray absorbing gas, but in others there is no clear physical correspondence between the UV and X-ray absorbing components.

\section{Acknowledgements}

I thank all the members of the FUSE AGN Working Group for their contributions to this research. This work is based on data obtained for the Guaranteed Time Team by the NASA-CNES-CSA FUSE mission operated by the Johns Hopkins University. Financial support to U. S. participants has been provided by NASA contract NAS5-32985.

\section{References}

Adelberger, K. L., et al. 2003, ApJ, 584, 45

Arav, N., Korista, K. T., \& de Kool, M. 2002, ApJ, 566, 699

Arav, N., et al. 2003, ApJ, 590, 174

Blandford, R, D., \& Begelman, M. C. 1999, MNRAS, 303, L1

Blustin, A., et al. 2003, A\&A, 403, 481

Brotherton, M., et al. 2002, ApJ, 565, 800

Cavaliere, A., Lapi, A., \& Menci, N. 2002, ApJ, 581, L1

Crenshaw, D. M., et al. 1999, ApJ, 516, 750

Crenshaw, D. M., et al. 2003, ApJ, 594, 116

Elvis, M. 2000, ApJ, 545, 63

Espey, B. R., et al. 1998, ApJ, 500, L13

Evans, I., et al. 1993, ApJ, 417, 82

Gabel, J., et al. 2003a, ApJ, 583, 178

Gabel, J., et al. 2003b, ApJ, 595, 120

George, I. M., et al. 1998, ApJS, 114, 73

Granato, G. L., et al. 2004, ApJ, 600, 580

Hutchings, J., et al. 1998, ApJ, 492, L115

Hutchings, J., et al. 2001, ApJ, 559, 173 
Kaspi, S., et al. 2002, ApJ, 574, 643

Königl, A., \& Kartje, J. F. 1994, ApJ, 434, 446

Kraemer, S. B., et al. 2000, ApJ, 535, 53

Kraemer, S. B., et al. 2001, ApJ, 551, 671

Kraemer, S. B., et al. 2002, ApJ, 577, 98

Kriss, G. A. 2001, in Mass Outflow in Active Galactic Nuclei: New Perspectives (eds. D. M.

Crenshaw, S. B. Kraemer, \& I. M. George) A. S. P. Conference Series, 255, 69

Kriss G. A., et al. 1992, ApJ, 392, 485

Kriss G. A., et al. 1995, ApJ, 454, L7

Kriss G. A., et al. 1996a, ApJ, 467, 622

Kriss G. A., et al. 1996b, ApJ, 467, 629

Kriss G. A., et al. 1997, in proc. IAU Colloquium 159 (ed. B. M. Peterson, F.-Z. Cheng, \& A.

S. Wilson) A. S. P. Conference Series, 113, 453

Kriss, G. A., et al. 2000a, ApJ, 535, 58

Kriss, G. A., et al. 2000b, ApJ, 538, L17

Kriss, G. A., et al. 2003, A\&A, 403, 473

Krolik, J. H., \& Kriss, G. A. 1995, ApJ, 447, 512

Krolik, J. H., \& Kriss, G. A. 2001, ApJ, 561, 684

Laor, A., et al. 1997, ApJ, 477, 93

Lee, J. C., et al. 2002, in X-ray Spectroscopy of AGN with Chandra and XMM-Newton (ed. T. Böller) (http://www.xray.mpe.mpg.de/ bol/agnspec/programm.html).

Mathur, S., Elvis, M., \& Wilkes, B. 1999, ApJ, 519, 605

Mathur, S., Wilkes, B., \& Elvis, M. 1995, ApJ, 452, 230

Mathur, S., Wilkes, B., Elvis, M., \& Fiore, F. 1994, ApJ, 434, 493

Moos, H. W., et al. 2000, ApJ, 538, L1

Murray, N., et al. 1995, ApJ, 451, 498

Miyaji, T., Wilson, A., \& Perez-Fournon, I. 1992, ApJ, 385, 137

Netzer, H., et al. 2003, ApJ, 599, 933

Ogle, P., et al. 2003, A\&A, 402, 849

Proga, D. 2000, ApJ, 538, 684

Reichard, T. A., et al. 2003, AJ, 125, 1711

Reynolds, C. S. 1997, MNRAS, 286, 513

Sako, M., et al. 2003, ApJ, 596, 114

Scannapieco, E. \& Oh, S. P. 2004, ApJ, submitted (astro-ph/0401087)

Scott, J. E., et al. 2004, ApJ, in press (astro-ph/0401226)

Silk, J., \& Rees, M. J. 1998, A\&A, 331, L1S

Telfer, R. C., et al. 2002, ApJ, 565, 773

Tolea, A., Krolik, J. H., \& Tsvetanov, Z. 2002, ApJ, 578, L31

Turner, T. J., et al. 2001, ApJ, 548, L13

Wyithe, J. S. B. \& Loeb, A. 2003, ApJ, 595, 614

Yaqoob, T., et al. 2003, ApJ, 582, 105

Zheng, W., et al. 1997, ApJ, 475, 469 